\title{
Effect of lightning induced voltage on the line polymer insulator in a distribution line
}

\begin{abstract}
Considering the performance of insulators versus lightning effects is an important issue for insulation coordination in distribution lines. In this paper, the performance of a polymer insulator $(33 \mathrm{kV})$ versus lightning induced voltage under different weather conditions was considered in transient domain and the profile of the voltage and fields along the insulator were evaluated and the results discussed accordingly. The results showed that the lightning induced voltage can have a great effect on the profile of the voltage and the fields along an insulator and also the weather conditions can profoundly affect the protection level of an insulator. Therefore, in order to set an appropriate protection level on the distribution line, consideration of the behaviour of an insulator under different weather conditions based on local data can be helpful.
\end{abstract}

Keyword: Electromagnetic fields; Induced voltage; Insulator; Lightning 